\title{
SERVICE DESK, POSSO AJUDÁ-LO? OU MELHOR, VOCÊ PODE ME AJUDAR?
}

\author{
Rosangela Klumb \\ rose.klumb@gmail.com \\ Universidade do Estado de Santa Catarina - Florianópolis, SC / Brasil \\ Francieli Murila Ferreira \\ fraaaancieli@gmail.com \\ Universidade do Estado de Santa Catarina - Florianópolis, SC / Brasil \\ Graziela Dias Alperstedt \\ gradial@gmail.com \\ Universidade do Estado de Santa Catarina - Florianópolis, SC / Brasil \\ Simone Ghisi Feuerschütte \\ simonegf@udesc.br \\ Universidade do Estado de Santa Catarina - Florianópolis, SC / Brasil
}

http://dx.doi.org/10.1590/1413-2311.0032013.40088

Recebido em 04/03/2013

Aprovado em 23/10/2013

Disponibilizado em 01/12/2014

Avaliado pelo sistema double blind review

Revista Eletrônica de Administração

Editor: Luís Felipe Nascimento

ISSN 1413-2311 (versão on-line)

Editada pela Escola de Administração da Universidade Federal do Rio Grande do Sul.

Periodicidade: Quadrimestral

Sistema requerido: Adobe Acrobat Reader.

\section{RESUMO}

O caso de ensino sobre a organização $X$ tem como objetivo oportunizar aos alunos a vivência de situações de conflito relacionadas à gestão da mudança, para a qual a etapa de planejamento foi pouco considerada, gerando um efeito cascata de resistências e de dificuldades. O caso ilustra um processo de mudança organizacional, seus limites e desafios considerando, em um primeiro momento, a inexistência de uma etapa de planejamento e, no segundo momento, a sua realização ao mesmo tempo em que eram sistematizadas as ações adotadas. Após um processo de reestruturação da Organização X, houve a implementação de uma nova ferramenta de Service Desk, amparada em processos de gerenciamento de serviços de Tecnologia da Informação (TI) e preconizada por um conjunto de melhores práticas de infraestrutura, operação e manutenção de serviços de TI, denominada ITIL - Information Technology Infrastructure Library. O objetivo dessa mudança era melhorar a qualidade da prestação dos serviços de TI na referida organização, de natureza pública, possibilitando, por meio de indicadores de eficiência, eficácia e efetividade, mensurar e avaliar a qualidade dos serviços de TI prestados a seus usuários. Como instrumento de ensino, o caso foi desenvolvido para ser usado em cursos de graduação e de pós-graduação nas áreas de Administração e Administração Pública, quando do debate em torno de temas como planejamento e gestão da mudança, desenvolvidos em disciplinas como Mudança Organizacional, Comportamento Organizacional, Planejamento Estratégico e Gestão de

REAd | Porto Alegre - Edição 79 - Nº 3 - setembro/dezembro 2014 - p. 823-837 
Service desk, posso ajudá-lo? Ou melhor, você pode me ajudar?

Pessoas. O caso também pode ser utilizado na discussão sobre a implementação de sistemas gerenciais no âmbito da Gestão de Sistemas de Informação e outras disciplinas da área de Tecnologia de Informação e Comunicação.

Palavras-chave: tecnologia da informação; gestão da mudança; resistência a mudanças; service desk.

\title{
SERVICE DESK, CAN I HELP YOU? OR BETTER, YOU CAN HELP ME?
}

\begin{abstract}
The teaching case on the organization $\mathrm{X}$ aims to create opportunities to pupils the experience of conflict related to change management, for which the planning stage was shortly considered, creating a ripple effect of resistance and difficulties. The case illustrates a process of organizational change, considering its limitations and challenges, at first, the absence of a planning step, and the second time, their achievement while they were systematized actions taken. After a restructuring of Organization X, was the implementation of a new Service Desk tool, supported by process management services for Information Technology (IT) and a set of recommended best practices for infrastructure, operation and maintenance services IT, called ITIL - Information Technology Infrastructure Library. The purpose of this change was to improve the quality of the provision of IT services in that organization, of a public nature, enabling, through indicators of efficiency, efficacy and effectiveness, measure and evaluate the quality of IT services provided to its users. As a teaching tool, the case was developed for use in undergraduate and graduate in the areas of Management and Public Administration, when the debate on topics such as planning and change management, developed in disciplines such as Organizational Change, Organizational Behavior, Strategic Planning and Personnel Management. The case can also be used in the discussion on the implementation of management systems within the Management Information Systems and other disciplines of Information Technology and Communication.
\end{abstract}

Keywords: information technology, change management, resistance to change, service desk.

\section{ESCRITORIO DE SERVICIO, COMO PUEDO AYUDAR? O MEJOR, USTED ME PUEDE AYUDAR?}

\section{RESUMEN}

El caso de la enseñanza en la organización X tiene como objetivo crear oportunidades a los alumnos la experiencia de los conflictos relacionados con la gestión del cambio, para el que la etapa de planificación fue poco considerada, creando un efecto dominó de resistencias y dificultades. El caso ilustra un proceso de cambio en la organización, teniendo en cuenta sus limitaciones y desafíos, en un primer momento, la ausencia de una etapa de planificación, y la segunda vez, su logro, mientras que las acciones fueron sistematizados adoptado. Después de una reestructuración de la organización $\mathrm{X}$, fue la implementación de una nueva herramienta de Service Desk, con el apoyo de los servicios de gestión de procesos de tecnología de la información (IT) y un conjunto de las mejores prácticas recomendadas para los servicios de infraestructura, operación y mantenimiento IT, llamado ITIL - Information Technology 
Rosangela Klumb, Francieli Murila Ferreira, Graziela Dias Alperstedt \& Simone Ghisi Feuerschütte

Infrastructure Library. El propósito de este cambio es mejorar la calidad de la prestación de servicios de TI en la organización de carácter público, lo que permite, a través de indicadores de eficiencia, eficacia y efectividad, medir y evaluar la calidad de los servicios de TI que presta a sus usuarios. Como una herramienta de enseñanza, el caso fue desarrollado para su uso en pregrado y postgrado en las áreas de Gestión y Administración Pública, cuando el debate sobre temas como la planificación y la gestión del cambio, desarrolladas en disciplinas tales como el cambio organizacional, Comportamiento Organizacional, Planificación Estratégica y Gestión de Personal. El caso también se puede utilizar en el debate sobre la aplicación de sistemas de gestión dentro de los Sistemas de Gestión de la Información y otras disciplinas de las Tecnologías de la Información y la Comunicación.

Palabras clave: tecnologías de la información; gestión del cambio; resistencia al cambio; mesa de servicio.

\section{INTRODUÇÃO}

Em meados do mês de dezembro de 2007, Carla ocupava a chefia da Seção de Patrimônio de Informática da Organização X, uma instituição de natureza pública com atuação jurisdicional no Estado de Santa Catarina, quando foi chamada para uma reunião com o seu Chefe, Coordenador de Infraestrutura, Sr. Paulo, e com o Secretário de Tecnologia da Informação, Sr. Rogério. Carla foi à reunião temendo que algo de errado pudesse ter ocorrido na execução das atividades na Seção que chefiava. Para sua surpresa, ambos a convidaram para assumir a Seção de Suporte, responsável pela prestação de suporte técnico de TI à aproximadamente 890 usuários. A Seção é formada por 20 colaboradores e tem muita visibilidade em função dos serviços executados. Silvia, que havia chefiado a área por oito anos, solicitou afastamento por motivos particulares. Carla sabia que a Seção de Suporte era uma das mais demandadas na Organização $X$, dela exigindo-se extrema pontualidade e qualidade de serviços, além de uma gestão de pessoas eficaz. Em termos financeiros a proposta não oferecia vantagens, no entanto, tratava-se de um desafio. Diante da oportunidade, Carla aceitou a proposta, sem saber das mudanças que estavam para acontecer no setor cuja chefia acabara de assumir.

\section{A ORGANIZAÇÃo}

A Organização X, objeto deste caso de ensino, é uma instituição pública sediada no Estado de Santa Catarina. A sua função jurisdicional está relacionada à resolução de conflitos 
Service desk, posso ajudá-lo? Ou melhor, você pode me ajudar?

de interesses, resguardando a ordem jurídica e a autoridade da lei. A atividade fim da Organização X é a produção de serviços à sociedade catarinense.

Para realizar suas atividades e atender suas funções, o órgão dispõe de equipes altamente capacitadas nas mais diversas áreas, incluindo: direito, administração, análise de sistemas, engenharia, entre outras; e conta em seu quadro com servidores concursados, servidores requisitados (servidores ou funcionários públicos das esferas federal, estadual ou municipal requisitados pela Organização X, na forma da lei, para o exercício de atividades de caráter técnico ou científico, típicas das suas unidades), técnicos terceirizados e estagiários. Além dos profissionais envolvidos na atividade fim, a Organização X conta com uma equipe de retaguarda que mantém todas as demais atividades consideradas meio.

Para efeitos deste caso de ensino e com o intuito de melhorar a compreensão do processo de implementação do Service Desk, apresenta-se parte da estrutura da Organização $\mathrm{X}$, conforme indicado na Figura 1:

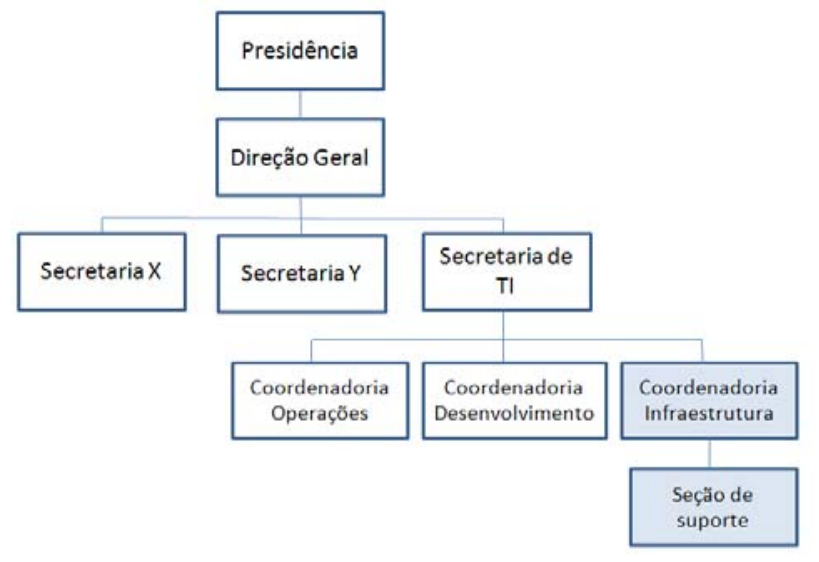

Figura 1 - Organograma da Organização X Fonte: elaborado pelas autoras

A Secretaria de Tecnologia da Informação (STI), macro unidade que integra a estrutura da Organização X e na qual foi implementado o gerenciamento de serviços de TI, é composta por três coordenadorias, quais sejam: Coordenadoria de Operações (CO), Coordenadoria de Desenvolvimento (CD) e Coordenadoria de Infraestrutura (CI), sendo cada uma desmembrada em seções. Os serviços da STI são voltados ao desenvolvimento de soluções corporativas e ao suporte técnico em TI, e prestados aos usuários internos da Organização X localizados na capital e no interior do Estado.

\section{A FERRAMENTA DE SERVICE DESK}

REAd | Porto Alegre - Edição 79 - Nº 3 - setembro/dezembro 2014 - p. 823-837 
Rosangela Klumb, Francieli Murila Ferreira, Graziela Dias Alperstedt \& Simone Ghisi Feuerschütte

Ao iniciar suas atividades na nova Seção, em janeiro de 2008, Carla recebeu a notícia de que havia sido adquirida uma nova ferramenta de Service Desk a ser utilizada por toda a Secretaria de TI do órgão, devendo o software ser parametrizado no prazo máximo de dois meses para atender às demandas da Seção e da Secretaria.

O Secretário de TI, Sr. Rogério, era uma pessoa visionária. Acreditava na inovação tecnológica como forma de solucionar muitos problemas e estava disposto a sempre implementá-la caso reconhecesse que a idéia era boa. Nesse período as melhores práticas da ITIL - Information Technology Infrastructure Library - já eram realidade em diversas empresas do setor privado e em organizações públicas.

A idéia inicial, segundo o Sr. Paulo, era a de contratar uma consultoria para implementar os processos de Gestão de Serviços de TI, preconizados pela ITIL. No entanto, como não havia orçamento disponível para a contratação de serviços de consultoria para auxiliar nesse trabalho, adquiriu-se a licença do software e o apoio da empresa contratada na implementação do mesmo. Juntamente com a disponibilização da ferramenta a empresa ministrou alguns treinamentos sobre a ITIL para os integrantes da organização.

Carla desconhecia as práticas da ITIL. Todavia, possuía experiência de dez anos em prestação de serviços de TI e realizava, na ocasião, o curso de graduação em administração pública, o que a ajudou neste desafio.

Gabriel, servidor que tomou posse junto com Carla na Organização X, era muito comprometido, dedicado e responsável, porém muito inseguro na tomada de decisão. Ele já trabalhava na Seção de Suporte quando Carla assumiu a chefia. Gabriel desempenhou um importante papel nesse processo de migração, auxiliando na parametrização da nova ferramenta.

O Sr. Paulo fazia muita pressão para que a ferramenta fosse logo implementada e, nessas circunstâncias, o software foi colocado em produção para uso em toda a Secretaria de TI, antecedido apenas de uma reunião com todos os colaboradores, a fim de orientá-los sobre as novas regras.

Juntamente com a ferramenta foram instituídos alguns conceitos relacionados ao processo de gerenciamento de serviços de TI - que eram novos para todos os envolvidos. O primeiro deles foi o processo de gerenciamento de incidentes, cujos responsáveis deveriam garantir o restabelecimento mais rápido possível dos serviços de TI em caso de indisponibilidade ou perda de qualidade do serviço. Para isso havia três níveis de atendimento: a) nível 1 - responsável pelo atendimento inicial, conforme procedimento

REAd | Porto Alegre - Edição 79 - N 3 - setembro/dezembro 2014 - p. 823-837 
Service desk, posso ajudá-lo? Ou melhor, você pode me ajudar?

descrito na Base de Conhecimento (base de dados com informações sobre a resolução dos diversos problemas existentes); b) nível 2 - equipe de suporte especializada nos diversos serviços que eram disponibilizados; e c) nível 3 - suporte realizado por fornecedores ou parceiros externos. Outra novidade foi o processo de gerenciamento de problemas, cuja atividade principal centrava-se na identificação da causa raiz dos incidentes que não possuíam uma solução conhecida.

Após a implementação da ferramenta de Service Desk e alinhada às boas práticas da ITIL, a Seção de Suporte passou a se denominar Central de Suporte.

\section{CONTEXTO DA IMPLEMENTAÇÃO}

Durante muito tempo os usuários dos serviços de TI da Organização X resolviam seus problemas com uma simples ligação para o técnico especializado. Este, por sua vez, mantinha o status de "especialista”, detendo o conhecimento exclusivo sobre determinados sistemas.

Todavia, a Central de Suporte tinha como uma de suas principais funções atuar como ponto único de contato entre os usuários de TI e a equipe técnica, desonerando os especialistas da atividade de suporte técnico e, dessa forma, permitindo que se dedicassem às atividades da seção a qual pertenciam e desenvolvessem projetos. Além disso, a Central de Suporte era responsável por manter uma base de conhecimento com a solução dos problemas rotineiros, que era compartilhada por toda a equipe.

Esse novo conceito de centralização dos serviços gerou muita resistência, principalmente por parte dos servidores públicos mais antigos, especialistas ligados à área de negócio do órgão. Um destes servidores antigos, que demonstrava resistência à mudança implementada, chegou a declarar: "é muito mais simples eu resolver diretamente com o usuário do que ter que passar tudo pela Central de Suporte”; "não há como padronizar o atendimento às solicitações da minha área, que são muito específicas”.

O primeiro aspecto mencionado pelos profissionais e que era foco da resistência, era a questão da usabilidade da Ferramenta de Service Desk. Os especialistas simplesmente se recusavam a utilizá-la e, dessa forma, impediam o fluxo do atendimento e uma resposta rápida ao usuário que estava com problemas.

Carla concordava com a melhoria gerada pela implementação da nova ferramenta, entretanto sentia-se desorientada na sua aplicação, conforme declarou: "Não havia envolvimento técnico de outros setores, como por exemplo, o do setor de planejamento

REAd | Porto Alegre - Edição 79 - N 3 - setembro/dezembro 2014 - p. 823-837 
Rosangela Klumb, Francieli Murila Ferreira, Graziela Dias Alperstedt \& Simone Ghisi Feuerschütte

estratégico e gestão de pessoas; havia apenas pressão pela implementação. Sentia-me cavando um túnel com colherinha de chá”.

Um ano após o início da implementação da ferramenta de Service Desk, o departamento de Carla ainda não havia alcançado, de modo concreto e verdadeiro, os indicadores de eficiência, eficácia e efetividade. As solicitações de suporte ainda não estavam sendo realizadas integralmente pela Central de Suporte e entre a equipe não havia um alinhamento das informações. Apesar de a ferramenta ser muito boa, era desacreditada por muitos usuários. Um usuário mais exaltado relatou certo dia à Carla: “Querida, eu entendo que devo usar a ferramenta, porém vocês mesmos não se entendem; dessa forma vou resolver as minhas solicitações como sempre fiz... esta ferramenta não funciona e nunca vai funcionar”.

A declaração deste funcionário representava o pensamento de muitos outros colaboradores da organização e tudo isso tinha fundamento. Hierarquicamente não havia sinergia sobre os resultados alcançados pela implementação da ferramenta. O Sr. Rogério, que respondia pela chefia da Secretaria de TI, tinha outros projetos nos quais estava focado, e dizia apenas que a ferramenta estava atingindo seus objetivos. Já o Sr. Paulo, responsável pela coordenação da Infraestrutura e chefe direto da Carla, percebia que a ferramenta não estava cumprindo suas finalidades, porém não tinha conhecimento técnico para auxiliar e nem influência para negociar recursos financeiros para contratar consultoria especializada.

\section{OS NOVOS GESTORES}

Em março de 2009 toma posse o novo presidente da Organização X. O então Secretário de TI (Sr. Rogério), por motivos pessoais, solicita exoneração da função comissionada que exercia. O Diretor Geral pede apoio ao Assessor de Planejamento, que já havia atuado na secretaria de TI e este sugere o nome do Sr. Rubens, analista de sistemas e lotado na Coordenadoria de Desenvolvimento, para exercer a função de Secretário de TI.

Com a nomeação do Sr. Rubens como Secretário de TI começa uma mudança interna, com a substituição dos coordenadores: o primeiro a ser substituído foi o Sr. Paulo, trocado pelo Sr. Elias, profissional formado em Engenharia de Produção e com experiência em infraestrutura tecnológica.

\section{O DESFECHO DO CASO}

REAd | Porto Alegre - Edição 79 - N 3 - setembro/dezembro 2014 - p. 823-837 
Service desk, posso ajudá-lo? Ou melhor, você pode me ajudar?

Nos dois anos seguintes à posse dos novos gestores algumas mudanças foram traçadas. Entre as principais medidas desenvolvidas estavam: a contratação de consultoria para modelagem dos processos ITIL; o desenvolvimento do Planejamento Estratégico de TI e; a adoção das boas práticas do COBIT para nortear a implementação da governança de TI na Organização X.

Uma vez contratada a consultoria, a primeira etapa realizada foi a modelagem de processos. Para tanto, constituiu-se uma equipe informal de quatro servidores coordenados por Gabriel que, juntamente com o apoio do consultor, realizaram a modelagem de cinco processos de gerenciamento dos serviços de TI em um período de seis meses. Este trabalho era acompanhado à distância por Carla. Como ela chefiava a seção não tinha como participar integralmente do mapeamento dos processos, que exigia muita dedicação. De qualquer forma, sempre que o consultor se dirigia à organização, ela participava das reuniões.

A contratação da consultoria especializada redirecionou a implementação da Ferramenta de Service Desk. A nova equipe que assumiu o processo conduziu o projeto com muito sucesso, alcançando todos os objetivos propostos inicialmente.

Paralelamente, foi elaborado o Planejamento Estratégico de TI da Organização X, alinhado ao Planejamento Estratégico Corporativo. O referido documento previa como meta principal que a Organização X se tornasse referência em Governança de TI dentro da sua área de atuação. Entre os principais objetivos a serem alcançados destacavam-se: a) aplicar os princípios de governança de TI por meio da implantação uma gestão de TI, em conformidade com as melhores práticas de governança, contribuindo para o alinhamento estratégico, a entrega de valor, a medição do desempenho e o gerenciamento de riscos e recursos; b) promover a melhoria contínua da qualidade dos serviços e soluções de TI, elevando continuamente o nível de atendimento às necessidades dos usuários, para que possam reconhecer a qualidade dos serviços e soluções oferecidos; e c) garantir a infraestrutura de TI, estruturando e gerenciando os recursos de TI de forma a garantir o pleno desempenho das atividades essenciais do órgão.

Em meio a este cenário, a Central de Suporte passou a ser designada de Central de Serviços de TI e, mais que um ponto de suporte aos usuários dos serviços de TI, tornou-se a principal interface operacional entre a área de TI e os usuários de seus serviços, sendo responsável pela primeira impressão que a área de TI transmite aos seus usuários. Dessa 
Rosangela Klumb, Francieli Murila Ferreira, Graziela Dias Alperstedt \& Simone Ghisi Feuerschütte

forma, o gerenciamento por processos foi introduzido na organização, possibilitando a melhoria contínua da qualidade dos serviços de TI prestados pela Central de Serviços.

Em 2011 a Central de Serviços de TI da Organização X foi regulamentada por meio de uma ordem de Serviço da Direção-Geral e, no final do mesmo ano, foi realizada a pesquisa de satisfação dos usuários quanto à qualidade dos serviços prestados pela Central de Serviços de TI. A avaliação teve como resultado a nota 8,77 (numa escala de zero a dez), o qual foi considerado muito bom pelos gestores da Organização X, considerando todos os percalços passados desde o início da implementação do Service Desk.

\section{ANEXO A - Notas de ensino}

\section{Objetivos educacionais do caso}

O caso de Implementação do Service Desk tem por objetivo oportunizar aos alunos a vivência de situações de conflito relacionadas à gestão da mudança, para a qual a etapa de planejamento foi pouco considerada, gerando um efeito cascata de falhas e dificuldades e um ambiente de trabalho conturbado, que sobrecarrega setores e/ou equipes. A proposta é fazer com que os estudantes se coloquem na posição dos gestores (da seção, da área e do próprio departamento, a Secretaria de TI) para interpretar a situação de conflito e, a partir disso, traçar possíveis caminhos que ajudem a reverter as inadequações relativas ao planejamento da mudança.

\section{Utilização recomendada}

Como instrumento de ensino, o caso foi desenvolvido para ser usado em cursos de graduação e de pós-graduação nas áreas de Administração e Administração Pública, quando do debate em torno de temas como planejamento e gestão da mudança, desenvolvidos em disciplinas como Mudança Organizacional, Comportamento Organizacional, Planejamento Estratégico e Gestão de Pessoas. O caso também pode ser utilizado na discussão sobre implementação de sistemas gerenciais no âmbito da Gestão de Sistemas de Informação e em outras disciplinas da área de Tecnologia de Informação e Comunicação.

\section{Fontes de obtenção dos dados do caso}

A construção do caso foi baseada em dados coletados a partir da observação participante e por meio de entrevistas realizadas com alguns atores envolvidos no processo de mudança da Organização X. 
Service desk, posso ajudá-lo? Ou melhor, você pode me ajudar?

\section{Relevância dos tópicos}

Atualmente se vive uma fase de desenvolvimento tecnológico e inovação organizacional intensa. Embora o uso de ferramentas tecnológicas ou de novos sistemas de informação e comunicação melhorem potencialmente as rotinas de trabalho, há uma preocupação crescente quanto a capacidade de muitas organizações em administrar a mudança.

O caso Implementação de Service Desk se mostra oportuno por enfocar o tema gestão de mudanças, pois revela como em uma mesma organização o mesmo projeto conduzido de forma diferente pode ter desfechos completamente distintos, ou seja, demonstra que a forma como são conduzidas as mudanças pode determinar o sucesso ou o fracasso de um projeto.

\section{Questões para discussão}

1. Em sua opinião, o que comprometeu a implementação do Service Desk na Organização X em sua primeira tentativa? Se você fosse um gestor, o que faria de diferente para conduzir o processo?

2. Quais medidas poderiam ter sido tomadas para evitar a resistência à mudança por parte dos usuários e por parte da equipe interna, cujos membros se denominavam “especialistas”?

3. Identifique aspectos positivos relacionados à resistência à mudança.

4. Por que a mudança se torna, freqüentemente, uma questão política nas organizações?

\section{Análise do caso e dinâmica de discussão}

Vamos simular que a alta direção da organização em questão contratou uma consultoria para diagnosticar as causas geradoras da dificuldade de implementação do Service Desk, considerando a primeira fase de implementação do projeto. O objetivo é identificar os aspectos positivos e negativos relacionados à mudança e traçar alternativas para potencializar os aspectos positivos e atenuar os aspectos negativos.

O professor poderá estimular que os alunos comparem este caso com outras mudanças organizacionais por eles vivenciadas, criando outras referências que sirvam de apoio para discussão do mesmo.

Orientações para a dinâmica de discussão:

a) Divida a classe em grupos de quatro a cinco integrantes.

b) Cada grupo deve se colocar no papel da consultoria e identificar os aspectos positivos e negativos relativos à mudança.

REAd | Porto Alegre - Edição 79 - Nº 3 - setembro/dezembro 2014 - p. 823-837 
Rosangela Klumb, Francieli Murila Ferreira, Graziela Dias Alperstedt \& Simone Ghisi Feuerschütte

c) Cada grupo deve desenvolver um conjunto de estratégias para atenuar os aspectos negativos e estimular os aspectos positivos da mudança.

d) Cada grupo terá 20 minutos para desenvolver uma lista de mudanças que pretende introduzir.

e) Ao final, cada grupo irá indicar um representante para descrever aos colegas o plano escolhido.

A ideia é que os alunos exercitem sua capacidade de programar mudanças de acordo com o cenário e o contexto em que estão inseridos.

\section{Padrão de respostas das questões:}

1. Em sua opinião, o que comprometeu a implementação do Service Desk na Organização $X$ em sua primeira tentativa? Se você fosse um gestor, o que faria de diferente para conduzir o processo?

Primeiramente, é necessário compreender a cultura da organização, dimensão que é a essência de uma organização. Para tanto, apreender como os valores inerentes a cada pessoa se definem quando ela se integra à organização e de que forma tais valores são construídos e reconstruídos à medida que são confrontados com os valores dos demais e daqueles que gerenciam a organização. Esta compreensão contribui para a condução das organizações em direção à realização dos seus objetivos, afirmam Ferreira et al (2006). Para Bergue (2010) desconsiderar a teia cultural de uma organização por ocasião da concepção de propostas de transformação e melhoria pode ser fator determinante do insucesso do propósito.

Há que se compreender que a configuração cultural da organização - que emerge essencialmente dos interesses e valores das pessoas - constitui elemento dinâmico da organização, que requer adaptações aos novos cenários (BERGUE, 2010). Ainda, o processo de mudança nos padrões culturais da organização que, em tese, não admite rupturas bruscas e tampouco autoritárias, mas transformações adaptativas operadas em vetores essenciais dessa estrutura constitui, segundo Bergue (2010), substancial desafio imposto ao gestor.

Bergue (2010, p. 69-70) destaca que um dos aspectos importantes a ser considerado em um processo de mudança - percebido pelos agentes como processos complexos, que percorrem caminhos tortuosos e, sobretudo, lentos - é o papel dos atores organizacionais, que devem estar totalmente envolvidos com o processo de transformação.

O interesse na mudança precisa, portanto, transcender o nível aparente e adentrar nos estratos ocultos das relações que estruturam a organização, buscando transformá-la efetivamente, reorientando-a segundo as exigências ambientais emergentes (BERGUE, 2010).

REAd | Porto Alegre - Edição 79 - N 3 - setembro/dezembro 2014 - p. 823-837 
Service desk, posso ajudá-lo? Ou melhor, você pode me ajudar?

Como gestor, verifica-se que uma das principais falhas na implementação do Service Desk foi desconsiderar a cultura organizacional, não identificar grupos potencialmente resistentes à mudança, não considerar a etapa de sensibilização e a capacitação das pessoas envolvidas no processo de mudança. Além desses aspectos, o fato de se implementar uma ferramenta sem que previamente se tenha realizado o mapeamento do processo envolvido pode ser considerada uma das razões para a falta de êxito do projeto na primeira tentativa. Por outro lado, verifica-se a falta de alinhamento da estratégia setorial com a estratégia organizacional.

Feuerschütte (1997, p.78) destaca que nos processos de mudança os valores introduzidos por pressão externa ou originários na própria organização são, por vezes, confrontados com a cultura organizacional. Nesses casos, em razão de modificações nas interações organizacionais, os valores e interesses compartilhados nos diversos níveis podem sujeitar-se a situações de convergência ou divergência, dependendo da forma como as propostas e objetos da mudança são apresentados pelos dirigentes e interpretados pelos integrantes da organização.

2. Quais medidas poderiam ter sido tomadas para evitar a resistência à mudança por parte dos usuários e por parte da equipe interna que se denominava “especialistas”?

Verifica-se, no caso da Organização X, que os aspectos relacionados à comunicação e à atuação de representantes da alta administração na condução do processo de inovação não foram considerados ao se efetuar a mudança. Sobre isso Bergue (2010) afirma que a resistência a mudanças precisa ser compreendida a partir de um sistema de resultados, uma vez que as pessoas, diante de uma situação de mudança, efetuam uma avaliação “que resulta num quantum de perda pessoal em relação ao espaço e patrimônio pessoal já conquistado [...] além de uma expectativa de perda futura frente ao que uma trajetória projetada reserva em termos de ganhos previstos de qualquer ordem” (BERGUE, 2010, p. 51).

O autor também aponta que outra dimensão de perda a ser considerada pelas pessoas como ensejadora de ações de resistência aos processos de mudança são os riscos de afetação das condições que conferem estabilidade (segurança) às pessoas em relação aos processos e ao ambiente de trabalho. Nesse aspecto, percebe-se que a resistência à mudança dos “especialistas” da Organização X se deu pelo fato de acreditarem que a sua estabilidade e o seu status, enquanto conhecedores profundos de determinadas áreas (especialistas), poderiam estar ameaçados.

REAd | Porto Alegre - Edição 79 - N 3 - setembro/dezembro 2014 - p. 823-837 
Rosangela Klumb, Francieli Murila Ferreira, Graziela Dias Alperstedt \& Simone Ghisi Feuerschütte

Considerando os aspectos relacionados à resistência à mudança verifica-se, no caso exposto, que um dos principais fatores causadores da resistência de alguns grupos de “especialistas" foi justamente a falta de um planejamento adequado do processo, que incorporasse um envolvimento maior das áreas afetadas, principalmente quanto aos benefícios que ocorreriam a partir da mudança. Segundo Bergue (2010), a resistência à mudança pode ser minimizada, ou mesmo suprimida, se o indivíduo ou o grupo ao qual ela se destina for capaz de constatar resultado positivo no balanço de perdas e ganhos decorrentes da inovação. No exemplo da Organização X, poderiam ter sido demonstrados aos “especialistas” os benefícios oriundos da transferência das atividades rotineiras das equipes especializadas para a equipe do Service desk, possibilitando aos “especialistas” maior tempo livre para o desenvolvimento de novos projetos.

3. Identifique aspectos positivos relacionados à resistência à mudança?

Chu (2004) destaca que a resistência à mudança é apontada como a grande culpada por atrasos, resultados negativos e até mesmo pelo fracasso de processos de transformação. Todavia, existem, conforme estudo realizado pela autora, aspectos positivos na resistência à mudança, cuja percepção varia de acordo com a experiência, a posição ocupada e o papel desempenhado pelo indivíduo no processo. Normalmente, indivíduos mais experientes em posições de comando e com papéis de destaque no processo, tendem a perceber com mais clareza as dimensões positivas relacionadas à resistência à mudança (CHU, 2004, p. 42).

Entre os aspectos positivos associados à resistência à mudança destacam-se: (1) contribuição para a identificação e tratamento de lacunas; (2) identificação dos riscos envolvidos; (3) estímulo à reflexão e discussão; (4) melhoria do relacionamento interpares; (5) aperfeiçoamento do processo de comunicação; (6) identificação das necessidades específicas de cada área; e (7) verificação do alinhamento da mudança com os objetivos estratégicos da organização. Conforme indicado por Chu (2004), aspectos positivos relacionados à resistência à mudança existem, todavia, a percepção comum ainda é de que a resistência é sempre negativa.

4. Por que a mudança se torna, freqüentemente, uma questão política nas organizações?

O poder tem um papel chave na determinação de muitos aspectos da vida organizacional (MORGAN, 2007, p.208) e desempenha um papel preponderante no 
Service desk, posso ajudá-lo? Ou melhor, você pode me ajudar?

delineamento das ações que visam à mudança, sendo instrumento para viabilizar a sua efetivação (FEUERSCHÜTTE, 1997).

Morgan (2007) destaca, citando as idéias de Aristóteles, que a política vem da diversidade de interesses. Ela é claramente manifestada nos conflitos e jogos de poder e nas intrigas interpessoais que resultam do fluxo da atividade organizacional e que ocorre em uma base contínua, muitas vezes, invisível para quase todos, exceto para os diretamente envolvidos (MORGAN, 2007).

Feuerschütte (1997), por sua vez, acrescenta que o funcionamento da organização resulta da relação entre o poder, enquanto dimensão política, e a cultura, a dimensão simbólica; ou seja, os mecanismos de poder, expressos no processo de mudança organizacional, originam-se dos padrões culturais vigentes. A autora destaca que as modificações estruturais são enfatizadas, nos momentos de disputa pelo poder, por representarem mecanismos de ascensão a posições privilegiadas e uma possível influência futura no processo decisório, na alocação de recursos e no acesso à rede de informação e de comunicação.

O poder nas organizações está associado, segundo Morgan (2007), à capacidade de exercer controle sobre os recursos. A cultura organizacional não se limita aos valores, às crenças, aos princípios e às normas da organização, mas é afetada pelos processos de disputas, de decisões e de influências em torno dos quais os indivíduos e os grupos se articulam, buscando a distribuição e a manutenção do poder. Nesse aspecto, a dimensão política propicia uma visão baseada no pressuposto de que a organização compreende redes de atores organizacionais com interesses diferentes e, muitas vezes, contraditórios, que lutam e se insurgem, de forma aparente ou encoberta, a fim de privilegiar alguns grupos de interesses, já que nas organizações existem grupos que controlam áreas significativas de poder (MORGAN, 2007).

Para Morgan (2007, p. 146), a maior parte das pessoas que trabalham em uma organização admite, na privacidade, que está cercada por formas de “arranjos”, através dos quais diferentes pessoas tentam ir ao encontro de interesses particulares. Segundo o autor, pode-se analisar a política organizacional de maneira sistemática, focalizando as relações entre interesses, conflito e poder.

Interesses tratam de um conjunto complexo de predisposições que envolvem objetivos, valores, desejos, expectativas e outras orientações e inclinações que levam o indivíduo a agir em uma e não em outra direção (MORGAN, 2007, P. 153). O conflito, por 
Rosangela Klumb, Francieli Murila Ferreira, Graziela Dias Alperstedt \& Simone Ghisi Feuerschütte

sua vez, surge sempre que os interesses colidem. Ele pode ser pessoal, interpessoal ou entre grupos rivais e coalizões. Pode ser construído dentro das estruturas organizacionais, nos papéis, nas atitudes e nos estereótipos, ou surgir em função de recursos escassos. Pode ainda estar explícito ou implícito. Qualquer que seja a razão e qualquer que seja a forma que assuma, a origem do conflito reside em algum tipo de divergência entre interesses percebidos e reais (MORGAN, 2007, P. 160). Já o poder é o meio através do qual os conflitos de interesses são, afinal, resolvidos.

Por fim destaca-se, que é obrigatório reconhecer que as tensões entre os interesses particulares e organizacionais promovem o incentivo para os indivíduos agirem politicamente (MORGAN, 2007).

\section{REFERÊNCIAS}

BERGUE, Sandro Trescastro. A redução sociológica na transposição de tecnologias gerenciais: gestão pela qualidade e certificação ISO 9001:2000 no Tribunal de Contas do Estado do Rio Grande do Sul. 2009. 329 f. Tese (Doutorado em Administração) - Programa de Pós-graduação em Administração, Universidade Federal do Rio Grande do Sul. Porto Alegre, 2009.

BERGUE, Sandro Trescastro. Cultura e mudança organizacional. Florianópolis: Departamento de Ciências da Administração/UFSC: UAB, 2010.

CHU, Rebeca Alves. Resistir é preciso. Revista de Administração Empresarial Executivo. São Paulo: FGV, v. 3, n. 1, fev/abr 2004, p. 41-45.

FERREIRA, Victor Claudio Paradela et al. Modelos de gestão. 2. ed. Rio de Janeiro: Ed. da FGV, 2006.

FEUERSCHÜTTE, Simone Ghisi. Cultura organizacional e dependências de poder: a mudança estrutural em uma organização do ramo de informática. Revista de Administração Contemporânea. v. 1, n.2, Maio/Ago. 1997, p.73-95.

MORGAN, Gareth. Imagens da organização: edição executiva. São Paulo: Atlas, 2007. 